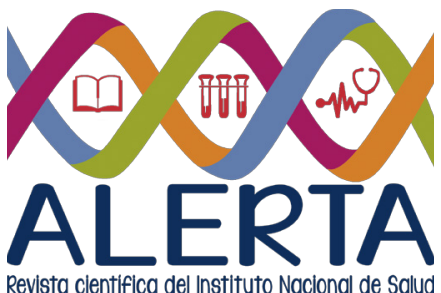

Revista cientifica del Instituto Nacional de Salud

\title{
Similitud de bases de datos de infecciones asociadas a atención sanitaria de hospitales de tercer nivel
}

\author{
José Eduardo Oliva ${ }^{1}$ \\ ${ }^{1}$ Instituto Nacional de Salud
}

Recibido: 2 de mayo de 2018 Aceptado: 4 de mayo de 2018 Correspondencia: joseduardoliva67@gmail.com

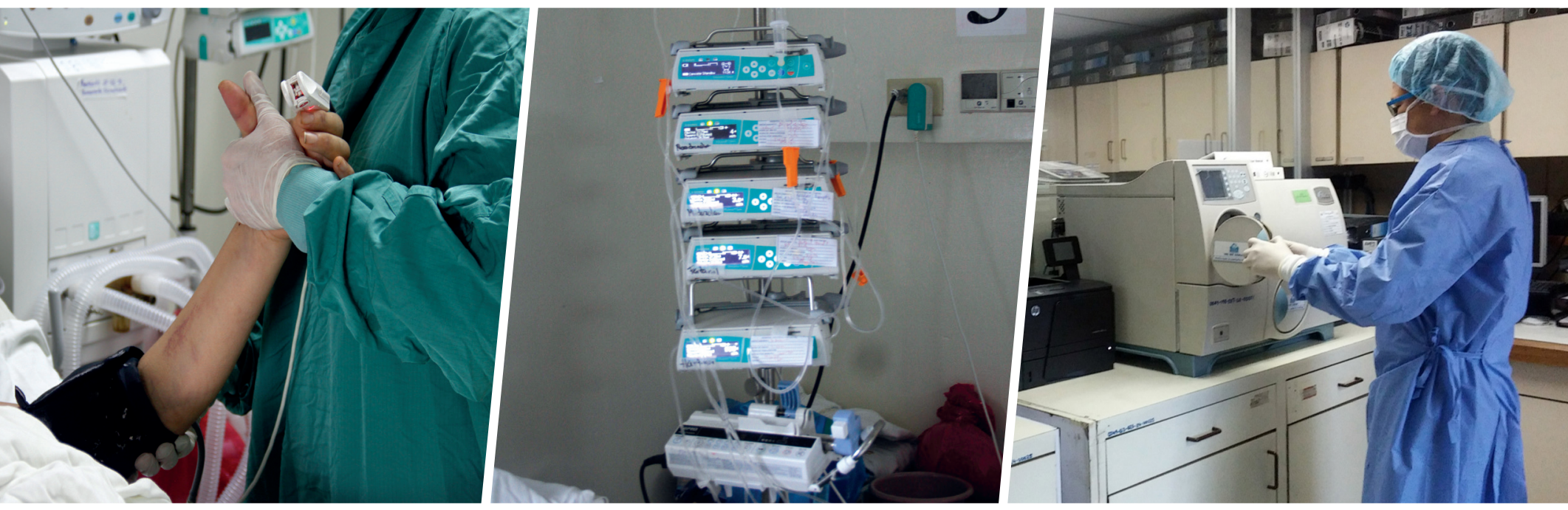

\section{Resumen}

Objetivo. Determinar el grado de similitud entre las bases de datos de infecciones asociadas a atención sanitaria diagnosticadas en hospitales de tercer nivel del Ministerio de Salud.

Metodología. Observacional, descriptivo, comparando retrospectivamente las bases de datos digitales y formularios físicos de recolección de información de las infecciones asociadas a atención sanitaria de los hospitales de tercer nivel, durante 2015. La similitud entre las bases se especificó en porcentajes. Resultados. El Hospital Nacional de Niños Benjamín Bloom y el Hospital Nacional Rosales no elaboraron formularios para Notificación Individual de Infecciones Asociadas a Atención Sanitaria durante 2015. Lo anterior podría deberse a que los clínicos responsables del llenado del Formulario para Notificación Individual de Infecciones Asociadas a Atención Sanitaria, como los clínicos responsables de registrarla en los Formularios de Ingreso/Egreso, no ejecutan la obligatoriedad de reportar toda infección asociada a atención sanitaria detectada. El Hospital Nacional de la Mujer, a través del Formulario para Notificación Individual de Infecciones Asociadas a Atención Sanitaria, reportó en el Sistema Nacional de Vigilancia Epidemiológica cerca del $80 \%$ de las infecciones asociadas a atención sanitaria reportadas por su laboratorio durante 2015. Conclusiones. La similitud entre bases digitales de datos de infecciones asociadas a la atención sanitaria, así como entre estas y los formularios que las alimentan, no es satisfactoria en el Hospital Nacional de Niños Benjamín Bloom y Hospital Nacional Rosales. En el Hospital Nacional de la Mujer es satisfactoria, exceptuando el traslado de datos de los Formularios de Ingreso/Egreso, al Sistema de Morbimortalidad en Línea.

Palabras claves: Infecciones asociadas a atención sanitaria, bases de datos, vigilancia epidemiológica.

\section{Abstract}

Objective. Determine the degree of similarity between Healthcare-Associated Infections databases, diagnosed in third-level hospitals of El Salvador's Ministry of Health.

Methodology. Observational, descriptive, comparing retrospectively HealthcareAssociated Infections digital databases and the data collection tools used to feed them, during 2015. The similarity between databases, and between these and the data collection tools used to feed them, was specified in percentages. Results. Hospital Nacional de Niños Benjamín Bloom and Hospital Nacional Rosales did not elaborate Forms for Individual Notification of Healthcare-Associated Infections during 2015. This could be due to the fact that clinicians responsible for filling out this form, as clinicians responsible for filling out the Admission/Discharge Form, do not comply to report all detected Healthcare-Associated Infections. Hospital Nacional de la Mujer, through the Form for Individual Notification of Healthcare-Associated Infections, reported in the Sistema Nacional de Vigilancia Epidemiológica database about $80 \%$ of the Healthcare-Associated Infections reported by their laboratory during 2015. Conclusions. The similarity between digital data bases of HealthcareAssociated Infections, and the similarity between these and data collection tools that feed them, is not satisfactory in Hospital Nacional de Niños Benjamín Bloom and Hospital Nacional Rosales. In Hospital Nacional de la Mujer the similarity is satisfactory, except for the transfer of data from the Admission/Discharge Form to the Sistema de Morbimortalidad en Linea database.

Keywords: Healthcare-Associated Infections, databases, epidemiologica surveillance. 


\section{Introducción}

Las Infecciones Asociadas a Atención Sanitaria (IAAS) plantean una considerable carga para la asistencia médica en todo el mundo. Aproximadamente 1.7 millones de personas desarrollan una IAAS en los Estados Unidos de América cada año. Alrededor de 4 millones de personas las presentan en la Unión Europea anualmente, de los cuales aproximadamente 37000 mueren a consecuencia directa de las mismas ${ }^{1,2}$. En México se observan tasas ligeramente mayores que en los estudios europeos ( $11.1 \%$ vs. $9.7 \%$, respectivamente) ${ }^{3}$.

La vigilancia y la retroalimentación de las IAAS han sido una piedra angular de los programas de prevención de infecciones desde los años 70, década en que las actividades de los programas de vigilancia se asociaron a una reducción en la incidencia de las mismas ${ }^{4}$. Los programas de vigilancia requieren tasas de IAAS fiables, entregadas de manera oportuna y eficiente.

Las grandes bases de datos se han vuelto una necesidad en salud pública, con lo que se vuelve imperativo verificar su calidad. Debido a los altos costos que involucra el revisar fuentes primarias de datos, la vigilancia electrónica emerge como alternativa para la evaluación de la calidad de los mismos ${ }^{5}$.

Una solución práctica al problema de mala calidad del dato es prevenir que sean ingresados datos erróneos a un sistema digital. Tomando en cuenta lo anterior, la atención debe ser dirigida no a los datos existentes en las bases, sino a los procesos que los introducen, modifican y transforman. Al ser estos procesos mejorados y controlados, se obtendrá una mejoría sostenible en la calidad del dato 6 .

Todo hospital debe contar con un Comité de Infecciones Asociadas a Atención Sanitaria (CIAAS) conformado según lineamientos internacionales ${ }^{7}$ : personal a tiempo completo incluyendo un coordinador, una secretaria, una enfermera por cada 250 camas y un técnico en saneamiento ambiental, así como una estrecha relación con la Unidad de Epidemiología del hospital.

El Ministerio de Salud, a través de la Dirección de Enfermedades Infecciosas, ha elaborado Lineamientos técnicos para la prevención y control de las infecciones asociadas a la atención sanitaria en 2010 y 20158,9. La implementación de la vigilancia electrónica de IAAS en el Sistema de Morbimortalidad en Línea (SIMMOW) y el Sistema Nacional de Vigilancia Epidemiológica (VIGEPES) se inició en el año 2013, al agregar las casillas correspondiente a IAAS en el Formularios de Ingreso/ Egreso (FIE) de cada paciente y con la obligatoriedad del llenado del Formulario para Notificación Individual de Infecciones Asociadas a Atención Sanitaria (VIGEPES 07). El VIGEPES 07 es elaborado por cada evento que presente un paciente durante su estancia hospitalaria. Posteriormente los datos contenidos en estos son digitados al VIGEPES. Así mismo, al ser dado de alta el paciente, se documenta en su Formulario de Ingreso/ Egreso (FIE), si presentó o no una IAAS durante su estadía en el hospital. Este formulario es digitado posteriormente en el SIMMOW.

Ambos sistemas permiten conocer si se presentan IAAS en los hospitales, el primero (VIGEPES) permite conocer la cantidad de infecciones que presenta un paciente durante su hospitalización y el segundo (SIMMOW), la cantidad de usuarios que se infectaron. El primero mide concentración y el segundo personas afectadas.

Las bases de datos de IAAS son heterogéneas, lo cual es evidente al revisarlas. EI FIE no es un formulario de notificación, refleja la existencia de una IAAS solo si el paciente la presenta durante su ingreso. En cambio, VIGEPES-07 sí es específico para notificación de caso; por ende, no serán congruentes, ya que sus objetivos son diferentes. Por lo anterior, la similitud entre SIMMOW y VIGEPES, bases digitales que se alimentan de los FIE y VIGEPES 07 respectivamente, tampoco será congruente en un $100 \%$. 
Sin embargo, tomando en cuenta que ambas herramientas de recolección de datos son elaboradas por los médicos tratantes durante y al final de la estancia hospitalaria de cada paciente, las bases de datos descritas deben contener datos parecidos, con una similitud satisfactoria.

El estudio tiene como propósito determinar el grado de similitud entre las bases de datos digitales de las infecciones asociadas a la atención sanitaria, diagnosticadas en los hospitales de tercer nivel del Ministerio de Salud, así como la similitud de estas bases de datos con los formularios físicos que las alimentan.

\section{Metodología}

Como marco de referencia, los tres hospitales evaluados son hospitales de tercer nivel y de referencia nacional. El Hospital Nacional de Niños Benjamín Bloom (HNNBB) cuenta con 396 camas y produce 12922 egresos cada año; el Hospital Nacional Rosales (HNR) cuenta con 694 camas y produce 22178 egresos cada año y el Hospital Nacional de la Mujer (HNM) cuenta con 422 camas y produce 21533 egresos cada año según datos del SIMMOW 2015.

Tipo de estudio observacional, descriptivo, comparando retrospectivamente las bases de datos digitales y formularios físicos de recolección de información que las alimentan, de las infecciones asociadas a atención sanitaria de los hospitales de tercer nivel de El Salvador, durante el año 2015. La similitud entre las bases se especificó en porcentajes.

Se realizó un muestreo aleatorio simple, partiendo de 2870 casos de IAAS registrados en el Cubo Bacteriológico durante 2015 (se incluyó un caso por paciente), el cual es un Sistema de Vigilancia Epidemiológica alimentado por los laboratorios locales de cada hospital.
Se consideró un nivel de confianza del 95\%, una prevalencia del $50 \%$ y un error de estimación del 7\%, obteniendo una muestra de 418 casos (HNNBB: 101, HNR: 155, HNM: 161).

Una vez obtenida la muestra, se hizo una comparación entre los casos de IAAS registrados en los laboratorios locales y aquellos registrados en las bases de datos VIGEPES, SIMMOW y CIAAS locales, así como entre estas y los formularios físicos (VIGEPES-07 y FIE) que las alimentan.

La similitud de información de registros de IAAS entre bases de datos digitales, y entre estas y los formularios físicos que las alimentan, se presentará en porcentajes, tomando como $100 \%$ los 418 casos de la muestra.

El protocolo de la investigación fue sometido a la evaluación del Comité Nacional de Ética de Investigación en Salud para su aprobación. Fue aprobado por escrito haciéndose constar en el acta: CORRES/CNEIS/143/201

\section{Resultados}

Tabla 1. Número consolidado de IAAS de los tres hospitales de tercer nivel durante 2015 según base de datos consultada

\begin{tabular}{|l|r|}
\hline BASES DE DATOS & Casos \\
\hline Laboratorios locales & 2870 \\
\hline CIAAS locales & 2687 \\
\hline VIGEPES & 1475 \\
\hline SIMMOW & 1312 \\
\hline
\end{tabular}

Fuente: Elaboración propia con bases de datos de laboratorio consolidadas en el Cubo Bacteriológico, CIAAS locales, VIGEPES y SIMMOW.

En la Tabla 1 se detalla el número de IAAS reportadas en los tres hospitales de tercer nivel del Minsal durante 2015, según la base de datos consultada. Es mayor el número de casos reportados por los laboratorios locales, con respecto a los encontrados en las otras bases de datos. Por lo anterior, los laboratorios se tomaron como punto de partida para calcular la muestra. 
Tabla 2. Consolidado de similitudes Hospital Nacional de Niños Benjamín Bloom

\begin{tabular}{|l|r|r|}
\hline HOSPITAL BLOOM & Casos & \% de similitud \\
\hline LAB/VIGEPES 07 & 101 & $32.7 \%$ \\
\hline LAB/FIE & 101 & $29.7 \%$ \\
\hline LAB/CIAAS & 101 & $32.7 \%$ \\
\hline LAB/SIMMOW & 101 & $28.7 \%$ \\
\hline LAB/ID VIGEPES & 101 & $0.0 \%$ \\
\hline VIGEPES 07/IDVIGEPES & 101 & $67.3 \%$ \\
\hline FIE/SIMMOW & 101 & $99.0 \%$ \\
\hline VIGEPES 07/FIE & 101 & $63.4 \%$ \\
\hline SIMMOW/VIGEPES & 101 & $71.3 \%$ \\
\hline
\end{tabular}

Fuente: Elaboración propia con bases de datos de laboratorio consolidadas en el Cubo Bacteriológico, CIAAS locales, expedientes clínicos, VIGEPES y SIMMOW.

Se partió de 101 expedientes evaluados de los 102 que se componía la muestra, ya que un expediente no contaba con el FIE correspondiente a la fecha de la IAAS reportada por el laboratorio local. Se analizaron en el orden que fueron comparadas, cada una de las bases de datos de esta institución y el Minsal.

\section{LAB/CIAAS/SIMMOW/IDVIGEPES}

Al investigar el porcentaje de similitud entre la base de datos del laboratorio local (estándar de oro) y el resto de bases digitales (CIAAS, SIMMOW, VIGEPES), se determinó que dicho porcentaje no alcanza el 33\% en ninguno de los casos, presentando una similitud del $32.7 \%, 28.7 \%$ y $0 \%$ respectivamente.

\section{LAB/FIE/VIGEPES 07}

Los mismos resultados se obtuvieron al comparar la base de datos del laboratorio local con los datos encontrados en las herramientas físicas que alimentan al SIMMOW y al VIGEPES, siendo estas el FIE con $29.7 \%$ y VIGEPES-07 con $32.7 \%$ de similitud, respectivamente.

\section{VIGEPES 07/IDVIGEPES}

Al investigar el porcentaje de similitud entre los formularios VIGEPES-07 elaborados y los casos reportados en el VIGEPES, se determinó que en el $67.3 \%$ de los casos ambas bases de datos concordaron en no registrar las IAAS reportadas por el laboratorio. Al 32.7\% restante les fue elaborado un VIGEPES-07 y su existencia verificada en los archivos del CIAAS local.

\section{FIE/SIMMOW}

El porcentaje de similitud entre las IAAS reportadas en los FIE y las IAAS reportadas en SIMMOW fue del 99\%. El porcentaje de similitud entre ambas bases demuestra que el traslado de la base física (FIE) a la base digital (SIMMOW) en esta institución es eficiente. Lo que menoscaba esta última aseveración es el hecho que solo el $29.7 \%$ de las IAAS de la muestra evaluada para esta institución fue reportado por el clínico responsable en los FIE elaborados al alta.

\section{VIGEPES 07/FIE}

El porcentaje de similitud entre las IAAS reportadas en los VIGEPES-07 y las IAAS reportadas en los FIE fue del $63.4 \%$.

\section{SIMMOW/IDVIGEPES}

Al investigar el porcentaje de similitud entre las dos bases digitales de datos nacionales (SIMMOW y VIGEPES), se determinó que estas concordaban en no registrar las IAAS reportadas por el laboratorio de la institución en el $71.3 \%$ de los casos. Es decir, en ambas bases de datos no se registró aproximadamente el $70 \%$ de casos de IAAS reportadas por el laboratorio local. 
Tabla 3. Consolidado de similitudes - Hospital Nacional Rosales

\begin{tabular}{|l|r|r|}
\hline HOSPITAL ROSALES & Casos & \% de similitud \\
\hline LAB/VIGEPES 07 & 155 & $0.0 \%$ \\
\hline LAB/FIE & 155 & $14.8 \%$ \\
\hline LAB/CIAAS & 155 & $3.9 \%$ \\
\hline LAB/SIMMOW & 155 & $12.3 \%$ \\
\hline LAB/ID VIGEPES & 155 & $0.0 \%$ \\
\hline VIGEPES 07/ID VIGEPES & 155 & $100.0 \%$ \\
\hline FIE/SIMMOW & 155 & $97.4 \%$ \\
\hline VIGEPES 07/FIE & 155 & $85.2 \%$ \\
\hline SIMMOW/ID VIGEPES & 155 & $87.7 \%$
\end{tabular}

Fuente: Elaboración propia con bases de datos de laboratorio consolidadas en el Cubo Bacteriológico, CIAAS locales, expedientes clínicos, VIGEPES y SIMMOW.

Se partió de 155 expedientes evaluados de los 155 que componía la muestra. Se analizaron en el orden que fueron comparadas, cada una de las bases de datos de esta institución y el Minsal.

\section{LAB/CIAAS/SIMMOW/IDVIGEPES}

Al investigar el porcentaje de similitud entre la base de datos del laboratorio local y el resto de bases digitales (CIAAS, SIMMOW, VIGEPES) se determinó que dicho porcentaje no alcanza el $15 \%$ en ninguno de los casos, presentando una similitud del $3.9 \%, 12.3 \%$ y $0 \%$ respectivamente. Por lo que durante el año 2015 el CIAAS local, el SIMMOW y el VIGEPES registraron menos del $15 \%$ de las IAAS reportadas por el laboratorio local.

\section{LAB/FIE/VIGEPES-07}

Los mismos resultados se obtuvieron al comparar la base de datos del laboratorio local con los datos encontrados en las herramientas físicas que alimentan al SIMMOW y al VIGEPES, siendo los FIE con $14.8 \%$ y los VIGEPES-07 con $0 \%$ de similitud, respectivamente.

\section{VIGEPES-07/IDVIGEPES}

Al investigar el porcentaje de similitud entre los formularios VIGEPES-07 elaborados y los casos reportados en el VIGEPES, se determinó que en el $100 \%$ de los casos ambas bases de datos concordaron en no registrar las IAAS reportadas por el laboratorio local.

\section{FIE/SIMMOW}

El porcentaje de similitud entre las IAAS reportadas en los FIE y las IAAS reportadas en SIMMOW fue del 97.4\%. El porcentaje de similitud entre ambas bases demuestra que el traslado de la base física (FIE) a la base digital (SIMMOW) en esta institución es excelente. Lo que menoscaba esta última aseveración es el hecho de que en el HNR solo el $14.8 \%$ de las IAAS reportadas por el laboratorio local son registradas por el clínico responsable en los FIE, elaborados al alta del paciente.

\section{VIGEPES-07/FIE}

Al investigar el porcentaje de similitud entre las IAAS reportadas en los VIGEPES-07 y las IAAS reportadas en los FIE se determinó que ambas concuerdan en el 85.2\% de los casos en no registrar las IAAS reportadas por el laboratorio de la institución. Es decir, en ambas bases de datos no se registra aproximadamente el $85 \%$ de los casos de IAAS reportadas por el laboratorio local.

\section{SIMMOW/IDVIGEPES}

Al investigar el porcentaje de similitud entre las dos bases digitales de datos nacionales (SIMMOW y VIGEPES), se determinó que estas concordaban en no registrar las IAAS reportadas por el laboratorio de la institución en el $87.7 \%$ de los casos. En otras palabras, en ambas bases de datos no se registra casi el $90 \%$ de los casos de IAAS reportadas por el laboratorio local del HNR. 
Tabla 4. Consolidado de similitudes - Hospital Nacional de la Mujer.

\begin{tabular}{|l|r|r|}
\hline MATERNIDAD & Casos & $\%$ de similitud \\
\hline LAB/VIGEPES 07 & 161 & $78.9 \%$ \\
\hline LAB/FIE & 161 & $83.9 \%$ \\
\hline LAB/CIAAS & 161 & $78.9 \%$ \\
\hline LAB/SIMMOW & 161 & $54.0 \%$ \\
\hline LAB/ID VIGEPES & 161 & $78.9 \%$ \\
\hline VIGEPES 07/ID VIGEPES & 161 & $100.0 \%$ \\
\hline FIE/SIMMOW & 161 & $66.5 \%$ \\
\hline VIGEPES 07/FIE & 161 & $71.4 \%$ \\
\hline SIMMOW/ID VIGEPES & 161 & $49.1 \%$ \\
\hline
\end{tabular}

Fuente: Elaboración propia con bases de datos de laboratorios locales, CIAAS locales, expedientes clínicos, VIGEPES y SIMMOW.

Se tomaron en cuenta 161 expedientes evaluados de los 161 que se componía la muestra. Se analizarán en el orden que fueron comparadas cada una de las bases de datos de esta institución y el Minsal.

\section{LAB/CIAAS/SIMMOW/IDVIGEPES}

Al investigar el porcentaje de similitud entre la base de datos del laboratorio local y el resto de bases digitales (CIAAS, SIMMOW, VIGEPES), se determinó que dicho porcentaje fue del $78.9 \%$, 54\% y $78.9 \%$, respectivamente. Por lo que durante el año 2015 el CIAAS del HNM y el VIGEPES reportaron aproximadamente el $80 \%$ de las IAAS reportadas por el laboratorio local. No así las IAAS registradas en el SIMMOW, las cuales superaron apenas el $50 \%$ de las reportadas por el laboratorio local. Tomando en cuenta que el $83.9 \%$ de las IAAS reportadas por el laboratorio local se encuentran en los FIE elaborados al alta, es el traslado de datos de esta herramienta física al SIMMOW lo que está fallando.

\section{LAB/FIE/VIGEPES-07}

Al comparar la base de datos del laboratorio local con los datos encontrados en las herramientas físicas que alimentan al SIMMOW y al VIGEPES, los porcentajes de similitud fueron: $83.9 \%$ y $78.9 \%$ para FIE y VIGEPES-07, respectivamente.

\section{VIGEPES 07/IDVIGEPES}

El porcentaje de similitud entre los VIGEPES 07 elaborados y las IAAS reportadas en VIGEPES fue de $100 \%$. Esto denota una perfecta transferencia de datos de la herramienta física a la base digital.

\section{FIE/SIMMOW}

El porcentaje de similitud entre las IAAS reportadas en los FIE y las IAAS reportadas en SIMMOW fue del $66.5 \%$. El porcentaje de similitud entre ambas bases demuestra que el traslado de la base física (FIE) a la base digital (SIMMOW) en esta institución no es satisfactorio. Analicemos este resultado en conjunto con los porcentajes de similitud entre LAB/SIMMOW y LAB/FIE, descritos en el primer y segundo párrafos del análisis del HNM, respectivamente.

\section{VIGEPES-07/FIE}

El porcentaje de similitud entre las IAAS reportadas en los VIGEPES-07 y las IAAS reportadas en los FIE fue del $71.4 \%$.

\section{SIMMOW/IDVIGEPES}

El porcentaje de similitud entre las IAAS reportadas en SIMMOW y las IAAS reportadas en VIGEPES fue de 49.10\%. Llama la atención que este porcentaje entre bases digitales, el cual no alcanza el 50\%, está muy por debajo del porcentaje de similitud entre las herramientas físicas que las alimentan, siendo este del $71.4 \%$. Si tomamos en cuenta que la similitud entre VIGEPES-07 y VIGEPES es del $100 \%$, el eslabón donde hay problemas es, como se detalló anteriormente, el traslado de información de los FIE al SIMMOW. 


\section{Discusión}

Durante el año 2015 no hubo similitud entre las bases de datos de los laboratorios locales y las bases de datos de los CIAAS locales del HNNBB y HNR, así como tampoco entre estas y las bases de datos SIMMOW y VIGEPES. Específicamente en este último, en ambas instituciones la similitud fue nula. No así en el HNM, donde la similitud entre las bases de datos del laboratorio local, CIAAS local y VIGEPES, fue de aproximadamente el $80 \%$.

No hubo similitud entre las IAAS reportadas en los VIGEPES-07 y las IAAS reportadas en los FIE en el HNNBB y el HNR. Se debe hacer hincapié en que, para ambos hospitales, en más del $85 \%$ de los casos no se registraron en ambas herramientas las IAAS reportadas por sus laboratorios locales. La similitud entre los VIGEPES-07 y los FIE en el HNM fue satisfactoria.

La similitud entre los FIE elaborados y las IAAS reportadas en SIMMOW, tanto para el HNNBB como para el HNR, fue excelente. Lo que refuerza esta aseveración es el hecho de que en el HNNBB las IAAS reportadas en los FIE no alcanzan el tercio de los casos reportados por el laboratorio y en el caso del HNR las IAAS reportadas en los FIE no alcanzan la sexta parte de los casos reportados por el laboratorio. Para el HNM, se reportaron en SIMMOW cerca del $65 \%$ de los casos reportados en los FIE. Si tomamos en cuenta que para este hospital la relación $\mathrm{LAB} / \mathrm{FIE}$ supera el $80 \%$, existe un problema en el traslado de datos de los documentos físicos FIE al SIMMOW.

Durante 2015, la similitud entre los VIGEPES-07 elaborados y las IAAS reportadas en VIGEPES, no fue satisfactoria en el HNNBB, mientras que en el HNR fue nula. En el caso del HNM, la similitud entre los VIGEPES 07 elaborados y las IAAS reportadas en VIGEPES, fue excelente.
En lo referente a la similitud entre las bases digitales SIMMOW y VIGEPES, en el caso del HNNBB y HNR, es nula, ya que ambas instituciones no reportaron casos de IAAS en VIGEPES durante 2015. En el caso del HNM la similitud entre ambas bases se aproximó al $70 \%$.

\section{Conclusiones}

No existe similitud entre las bases de datos de los laboratorios locales y las bases de datos de los CIAAS del HNNBB y HNR, así como tampoco entre estas y las bases de datos de SIMMOW y VIGEPES. Lo anterior podría deberse a que los clínicos responsables del llenado del VIGEPES-07, al diagnosticar la IAAS, como los clínicos responsables de registrarla en los FIE, al dar el alta al paciente, no están ejecutando la obligatoriedad de reportar toda IAAS detectada en la institución.

En el HNM la similitud entre las bases de datos de laboratorio, CIAAS local y VIGEPES, fue satisfactoria. En el caso del SIMMOW, el porcentaje de similitud fue menor, ya que se reportaron en esta base digital cerca de la mitad de los casos reportados por el laboratorio. Si tomamos en cuenta que para este hospital la relación LAB/FIE superó el $80 \%$, existe un problema en el traslado de datos de los documentos físicos FIE al SIMMOW.

No se encontró similitud en el registro de la información de pacientes con IAAS. Los hallazgos sugieren que puede haber dificultad en el reporte de la información en y a partir de los formularios físicos de recolección. Se sugiere la implementación de una evaluación amplia del proceso de cada una de las etapas del registro de los casos de pacientes con IAAS.

\section{Agradecimientos}

Se agradece a los Directores, Comités de IAAS, Unidades de Epidemiología y al personal de ESDOMED del HNNBB, HNR y HNM, por facilitar las condiciones para la realización del estudio. 


\section{Conflicto de intereses}

El autor expresa no contar con conflictos de intereses; los fondos de la investigación fueron del Ministerio de Salud de El Salvador.

\section{Referencias bibliográficas}

1. Klevens RM, Edwards JR, Richards CL Jr, et al. Estimating health care associated infections and deaths in U.S. hospitals, 2002. Public Health Rep. 2007; 122:160-6.

2. European Centre for Disease Prevention and Control. Annual epidemiological report on communicable disease in Europe 2008. Stockholm: European Centre for Disease Prevention and Control; 2008.

3. Ponce de León-Rosales SP, Molinar-Ramos F, DomínguezCherit G, RangelFrausto MS, Vázquez-Ramos VG. Prevalence of infections in intensive care units in Mexico: a multicenter study. Crit Care Med. 2000 May; 28(5):1316-21.

4. Gaynes R, Richards C, Edwards J, et al. Feeding back surveillance data to prevent hospital-acquired infections. Emerg Infect Dis 2001; 7:295-8.

5. Roos L. Assessing Data Quality: A computarized approach. Soc Sci Med 1989; 28(2): 175-82.

6. Hub Y. Data Quality. Information and Software Technology 1990; 32(8): 559-65.
7. World Health Organization, Regional Office for the Western Pacific, World Health Organization, Regional Office for SouthEast Asia. Practical guidelines for infection control in health care facilities. [Internet]. Manila; New Delhi: World Health Organization, Regional Office for Western Pacific; World Health Organization, Regional Office for South-East Asia; 2004 [citado 7 de enero de 2018]. 110 p. (SEARO Regional publication). Disponible en: http://www.wpro.who.int/publications/docs/ practical_guidelines_infection_control.pdf

8. Ministerio de Salud de El Salvador. Lineamientos para el control de infecciones en la atención sanitaria. Primera edición noviembre de 2010. [Internet] El Salvador: Minsal; 2010[citado 17 enero 2018]. 159 pág. Disponible en: http://asp.salud.gob. sv/regulacion/pdf/lineamientos/lineamientos_infecciones_ atencion_sanitaria.pdf

9. Ministerio de Salud de El Salvador. Lineamientos técnicos para la prevención y control de las infecciones asociadas a la atención sanitaria. Segunda edición diciembre de 2015. [Internet]. El Salvador: Minsal; 2010 [citado 17 enero 2018]. 138 pág. Disponible en:http://asp.salud.gob.sv/regulacion/pdf/ lineamientos/lineamientos_tecnicos_infecciones_asociadas_ atencion_sanitaria.pdf

\section{Forma recomendada de citar}

Oliva JE. Similitud de base de datos de infecciones asociadas a atención sanitaria de hospitales de tercer nivel. Revista ALERTA. 2018;1(1):53-60. 